\title{
LA GIUSTIZIA ITALIANA NELLO SPECCHIO DELLE SCIENZE SOCIALI
}

\author{
Nota del s.c. VINCENZO FERRARI (*)
}

(Adunanza del 26 maggio 2011)

SUNTO. - In questo articolo vengono presentati succintamente i risultati di una vasta ricerca su "L'amministrazione della giustizia nell'Italia del 2000", svolta da un gruppo di studiosi di scienze sociali, sotto il coordinamento dell'autore, nella prima metà dello scorso decennio. L'indagine, da cui sono usciti dodici studi monografici raccolti in nove volumi, ha preso in considerazione il sistema della giustizia sia nella sua struttura e nelle sue funzioni, sia nei suoi rapporti con alcuni altri sistemi d'azione sociale, e precisamente la famiglia, la politica, il contesto europeo e l'avvocatura. L'autore mette in risalto la relativa chiusura della magistratura verso la società e le principali disfunzioni della giustizia civile e penale. Osserva poi che la congestione sembra un tratto comune a molti sistemi di giustizia moderni e, rifacendosi a recenti studi americani, affaccia l'ipotesi che tali sistemi riescano ad operare soltanto in presenza di alternative semi-istituzionali, come il patteggiamento nel penale e la negoziazione nel civile, che ne tradiscono i principi ispiratori.

ABSTRACT. - This article offers a brief description of an extensive research on "Justice in Italy in the 2000", conducted in the first half of the last decade by a group of social scientists led by the author. This project, whose results have been collected in nine volumes containing twelve monographic studies, focused on the structure and functions of the system of justice, as well as on how it relates to certain other systems of social action, such as the family, politics, the European context and the Bar. The author portrays the

(*) Università degli Studi di Milano. E-mail: vincenzo.ferrari@unimi.it 
Italian judiciary as relatively closed towards society and highlights the main dysfunctions of both civil and penal proceedings. He adds that congestion seems to be a common feature of many modern justice systems and, echoing some recent American studies, lays down the hypothesis that they only succeed in functioning when semi-institutional alternatives are also available, even if prima facie incompatible with their basic principles, such as plea bargaining in criminal and negotiation in civil proceedings.

Con questo intervento mi propongo di riferire a grandi linee dei risultati di una complessa ricerca su "L'amministrazione della giustizia nell'Italia del 2000", svoltasi nella prima metà dello scorso decennio in base a un progetto di fattibilità commissionato dal Consiglio Superiore della Magistratura al Centro nazionale di prevenzione e difesa sociale (CNPDS) e di un susseguente progetto esecutivo finanziato dal Ministero dell'Università e della Ricerca Scientifica.

Il tema, oggetto di infinite discussioni da decenni e affrontabile da diverse prospettive, è stato analizzato in questa indagine dal punto di vista delle scienze sociali, con l'intento di scoprire, attraverso l'osservazione empirica e il riferimento alle ipotesi teoriche più aggiornate, almeno alcune fra le cause di una crisi che perdura sin dall'unificazione del nostro paese. Al progetto, che ho coordinato attraverso l'Istituto di filosofia e sociologia del diritto dell'Università di Milano, hanno partecipato numerosi studiosi, prevalentemente sociologi del diritto, ma anche sociologi generali, economisti ed esperti di organizzazione. Ne sono usciti dodici studi monografici, quattro dei quali raccolti in un volume collettaneo a cura di Luigi Cominelli, che ha svolto centralmente attività di segretariato scientifico e organizzativo. Altri tre, pur compiuti e discussi come i precedenti in due convegni svoltisi nel 2004, non sono mai pervenuti nella forma definitiva, se si esclude un saggio che affrontava un aspetto parziale e che apparve, primo fra tutti, già nel 2002. L'attesa di questi lavori, protrattasi per anni, ha ritardato oltre misura la stesura del volume con cui, da coordinatore centrale, mi ero riproposto di concludere l'importante iniziativa. Quando (sperabilmente) comparirà, assumerà la ricerca come punto di partenza per ulteriori e più aggiornate riflessioni.

Il titolo dell'indagine, inizialmente ispirata al CSM da Eligio Resta, non fu scelto a caso. Infatti all'inizio degli anni ' 60 , e con gli stessi intenti, il CNPDS, diretto da Adolfo Beria di Argentine, aveva raccolto un gruppo di giuristi, sociologi, storici ed economisti, col compi- 
to di svolgere un'indagine intitolata "L'amministrazione della giustizia nella società italiana in trasformazione", da cui scaturirono tredici volumi pubblicati per le edizioni Laterza. Il coordinamento fu affidato a Renato Treves, che sintetizzò i risultati in un libro ampiamente diffuso, ${ }^{1}$ secondo una chiave interpretativa prevalentemente di sociologia del diritto. L'ideale collegamento con quel lontano progetto fu dunque spontaneo, e non solo per le coincidenze - il CNPDS, la sociologia del diritto milanese - ma anche per la necessità, teorica, storica e persino politica, di registrare omologie e discrepanze fra i due periodi, lontani fra loro ma accomunati, fra l'altro, proprio dall'insoluto stato di sofferenza del sistema della giustizia.

Perché questa sofferenza perdura nei decenni a dispetto di ogni intervento? Perché il nostro paese, che ha elaborato una dottrina giuridica tra le più fini del mondo, deve essere additato all'opinione pubblica internazionale come un luogo in cui è quasi impossibile ottenere giustizia, con le inevitabili ricadute anche sul piano economico? Perché nessun regime politico, dal centro-sinistra riformatore degli anni sessanta sino ai governi della cosiddetta Seconda Repubblica, è riuscito a venire a capo di questa situazione? Si tratta di una crisi endemica? E, infine, la crisi è un fatto esclusivamente italiano, o nel nostro paese viene soltanto amplificata?

Naturalmente il gruppo di lavoro che nel 2002 si accinse a realizzare il progetto non ambiva a rispondere compiutamente a queste domande, radicate e quasi incancrenite in 150 anni di storia patria. Il massimo cui poteva aspirare era far luce con una metodologia prevalentemente empirica su alcuni aspetti di quel complesso di azioni sociali che si svolgono, per dirla con Pierre Bourdieu, sociologo francese, nel "campo giuridico", di cui la giustizia dei tribunali rappresenta il nucleo più profondo, proprio perché pronuncia una parola vincolante sul diritto - ius dicit - passando dall'astratto degli enunciati normativi generali al concreto della sentenza.

Con questi intenti, la ricerca è stata impostata guardando all'amministrazione della giustizia come un complesso sistema d'azione sociale, visto sia nella sua struttura e nelle sue funzioni, sia nei suoi rapporti con altri sistemi d'azione sociale.

1 Treves R. Giustizia e giudici nella società italiana. Problemi e ricerche di sociologia del diritto. Laterza, Bari, 1972 (2a ed. 1975). 
Su questo punto premetto che questa opzione teorica non implicava - né ha implicato, nelle posizioni dei singoli studiosi - un'adesione alle correnti dello struttural-funzionalismo sociologico nelle sue diverse esplicazioni. L'orientamento sistemico è bensì comune a tutte le scienze sociali, per non dire a tutte le scienze, per l'essenziale ragione che quanto accade nel mondo non può essere scientificamente indagato isolatamente, procedendo per compartimenti stagni: infatti, come si dice efficacemente in tedesco, Alles ist mit Allem verbunden, ogni cosa è collegata con tutto il resto. Partendo da questa basilare osservazione, che orienta soprattutto il metodo d'indagine, si possono però costruire visioni del mondo fra loro ben diverse, di cui lo struttural-funzionalismo, o il funzionalismo strutturale come si definisce oggi, è solo una, apprezzabile per il respiro teorico, ma non necessariamente condivisibile, anzi criticabile proprio dal punto di vista della necessità di esaminare i nessi connettivi che collegano fra loro i diversi elementi di un sistema: la cui stessa identificazione, con i relativi confini, è largamente il frutto di scelte artificiali dettate dalla necessità di dotarsi di una semantica comune agli studiosi, per facilitarne la comunicazione. Soprattutto nell'ambito delle relazioni umane i sistemi non sono - come usa dire - objets trouvés, che il ricercatore trova sul suo cammino, ma costruzioni teoriche e simboliche utili al fine di osservare gli eventi, comprenderli e classificarli. Lo struttural-funzionalismo sociologico, al contrario, ha quasi ipostatizzato i sistemi come fossero entità reali, non ideali, dotate di vita propria, organica, assegnando loro funzioni adeguate all'equilibrato e ordinato svolgimento di tale vita. Questa prospettiva ha attirato su di sé forti critiche soprattutto nei momenti di tensione sociale, quando gli squilibri, piuttosto che l'equilibrio, destano l'interesse scientifico, e naturalmente sollecitato ricostruzioni e riformulazioni. Fra queste, la grande teorizzazione operata da Niklas Luhmann negli ultimi decenni del secolo passato è l'esempio più rimarchevole, ma neppure questa, pur ispirata ad una visione mobile dei sistemi e della loro relazione con l'ambiente circostante, è riuscita almeno ad avviso di chi scrive - a sottrarsi alle ipostatizzazioni e agli apriorismi dei predecessori.

Fatta questa premessa, succinta ma necessaria, passo a descrivere l'impianto generale della ricerca.

Il sistema della giustizia, come dicevo, è stato esaminato (per comodità) dapprima in sé, nella sua struttura e nelle sue funzioni, e successivamente nei suoi rapporti con altri sistemi d'azione sociale. 
Il primo aspetto ha preso in esame: (a) la magistratura italiana, nella sua composizione e nei suoi orientamenti; (b) la giustizia civile e le sue alternative; (c) la giustizia penale e le sue alternative. Il secondo aspetto ha preso in esame: (a) la magistratura nell'immagine pubblica; (b) la famiglia nell'opinione dei giudici; (c) la giustizia italiana nel contesto europeo; (d) il sistema della giustizia nei suoi rapporti con l'avvocatura.

Ognuno di questi aspetti si è avvalso di specifiche ipotesi teoricoempiriche. Si può però dire che un'ipotesi generale del lavoro, rimasta sullo sfondo, quasi come retro-pensiero del coordinatore, è stata quella, già emergente dalla ricerca Treves, e sostanzialmente confermata da altre ricerche apparse negli ultimi decenni, secondo cui la crisi della giustizia italiana è davvero endemica, certo influenzata da deficienze funzionali mai risolte (e forse neppure seriamente affrontate), ma dipendente da fattori più profondi, risalenti a un modo di concepire il rapporto di cittadinanza e lo stesso ordinamento giuridico. Una più compiuta riflessione, in tempi recentissimi, mi ha portato a rafforzare questa ipotesi anche alla luce di osservazioni comparative.

Procedo ora a descrivere succintamente i risultati ottenuti.

Come accennato, il primo tema affrontato è stato quello della magistratura, di cui la ricerca fornì subito, per dir così, una fotografia, aggiornata al 2000 (dato disponibile all'epoca), quindi ormai datata, ma in realtà non molto dissimile dalla situazione che emerge da rilevazioni recenti. Questa indagine pilota, svolta da Fabio Quassoli e Sonia Stefanizzi, ricercatori dell'Università di Milano-Bicocca, apparve già nel $2002^{2}$ e fornì all'intero gruppo un quadro strutturale essenziale per il prosieguo dei lavori. All'epoca i magistrati ordinari in servizio attivo nel paese erano 8.661, di cui 5.606 (64,7\%) maschi e 3.055 (35,3\%) femmine. Rispetto al 1991, preso in esame come punto di riferimento temporale, si era avuto un aumento complessivo dell' $8,12 \%$, non troppo modesto in sé e di significativo interesse quanto alla composizione di genere, giacché le percentuali rispettive di maschi e femmine equivalevano, un decennio prima, al 76,5\% e al 23,5\%. Di questo gruppo professionale, i ricercatori rimarcarono che era rimasto elitario malgrado il

2 Quassoli F., Stefanizzi S. I magistrati italiani. Un'analisi esplorativa delle caratteristiche socio-demografiche e dei percorsi di mobilità. Sociologia del diritto, XXIX, 2002, 1, pp. 89 ss. 
maggior livello di egualitarismo acquisito nei decenni dalla società italiana, riscontrarono la provenienza geografica (in maggioranza ancora meridionale) ed esaminarono alcune dinamiche interne, relative soprattutto ai trasferimenti e agli accessi alle cariche direttive.

Limiti di tempo impediscono di scendere più nei dettagli. Tuttavia, è interessante osservare che i dati più recenti reperibili presso il Consiglio Superiore della Magistratura confermano il trend allora registrato. I magistrati ordinari attualmente in servizio sono infatti 8.994, di cui 4.778 uomini $(53,12 \%)$ e 4.216 donne $(46,87 \%)$. A fronte di un aumento complessivo più modesto (vengono del resto denunciate 1.247 scoperture d'organico) appare ancora aumentato l'elemento femminile, entrato in magistratura negli anni sessanta e ormai prossimo al sorpasso. A parte questo aspetto di sicura importanza, il dato complessivo segnala che la consistenza numerica della magistratura non è trascurabile, considerando anche la sensibile presenza di magistrati onorari (più di 4.000 secondo le rilevazioni più recenti). Ovvero, pur tenendo conto dell'aumento (modesto) della popolazione residente e quello (assai più sensibile) del carico di lavoro, non è probabilmente nella scarsità di giudici e pubblici ministeri che va cercata la causa principale degli alti livelli di inefficienza che si riscontrano in molti uffici giudiziari. Un'osservazione, questa, confortata dalla comparazione con altri sistemi giudiziari. ${ }^{3}$ Altro discorso riguarda l'irrazionale distribuzione dei magistrati sul territorio, nei numerosi uffici giudiziari, fra i quali si osservano gravi squilibri, e l'endemica, mille volte denunciata, carenza di risorse e di infrastrutture.

Dai sociologi dell'Università di Milano-Bicocca, sotto la guida di Antonio De Lillo, fu aperto un capitolo a sé per studiare gli orientamenti ideologici dei magistrati italiani, secondo schemi di interpretazione consolidati in sociologia. Con metodo quantitativo, è stata svolta un'indagine mediante questionario somministrato a un campione di 1.131 magistrati, onde rilevarne notizie e opinioni su aspetti significativi della professione e della società. Con metodo qualitativo, è stata poi studiata l'attività di tre procure della Repubblica (Varese, Verona e Bergamo) nei loro rapporti col contesto sociale circostante.

3 Cfr. Systèmes judiciaires européens. Efficacité et qualité de la justice. Rapporto della Commissione europea per l'efficacia della giustizia (CETEJ, Consiglio d'Europa), Edizione 2010 (dati del 2008). 
La prima indagine 4 si è occupata essenzialmente di rilevare un autoritratto della magistratura ordinaria, ricorrendo a una tipologia proposta da Carlo Guarnieri, che ha distinto fra quattro diverse concezioni del suo ruolo sociale: il magistrato inteso come passivo "esecutore" della volontà del legislatore, come "delegato", operante in "una situazione di creatività giurisprudenziale controllata", come "guardiano", ovvero "custode dei diritti fondamentali del cittadino", e, infine, come "politico", protagonista della vita democratica non solo attraverso sentenze "creative", ma anche attraverso l'attivo intervento nella vita sociale. ${ }^{5}$ Accanto a ciò, sono stati rilevati gli orientamenti di valore dei magistrati su una scala comunemente in uso negli studi sociologici sulle professioni, onde valutare se la magistratura si caratterizzasse per una "cultura di ceto". Dai risultati è emerso che, fra i quattro modelli indicati, la grande maggioranza degli intervistati ha optato per quelli intermedi ("delegato" e "guardiano"), con qualche interessante oscillazione emergente dallo studio di alcune variabili (sesso, età, provenienza familiare, funzioni giudicanti o requirenti). Le reazioni degli intervistati ai numerosi items concernenti i valori sono state coerenti con questa concezione - diremo così - tradizionale della categoria, rispettosa di principi generali quali l'equità, la giustizia, l'autonomia, la tutela della socialità e dell'ambiente, e meno attratta da prospettive individualistiche o edonistiche. Complessivamente la magistratura è apparsa articolata al proprio interno, ma sempre coesa. La sua composizione è mutata senza troppo incidere sul suo carattere elitario.

La seconda indagine, ${ }^{6}$ svolta attraverso l'analisi congiunta di tre databases (statistiche giudiziarie, interviste semi-strutturate a procura-

4 Questa duplice ricerca è rimasta allo stato di rapporto, divulgato nel corso di due convegni, svoltisi nel 2004, in cui i risultati dell'indagine furono discussi. Cfr. De Lillo A., Quassoli F. \& Stefanizzi S. I magistrati italiani tra conservazione e mutamento. Primi risultati di una survey nazionale sulla magistratura italiana, Centro nazionale di prevenzione e difesa sociale. Milano, 2004 (non pubblicato).

5 Guarnieri C. Giustizia e politica. I nodi della Seconda Repubblica. Il Mulino, Bologna, 2003.

6 Quassoli F., Stefanizzi S. (a cura di), con la collaborazione di Niessen B, Poletti C. e Tosi S. La costruzione sociale delle "politiche giudiziarie": cultura organizzativa, ideologia professionale e comunicazioni con l'esterno in tre Procure della Repubblica dell'Italia settentrionale. Centro nazionale di prevenzione e difesa sociale. Milano, 2004 (non pubblicato). 
tori e sostituti procuratori della Repubblica, articoli apparsi sui media sull'arco di tre anni), ha messo in luce soprattutto un aspetto di cruciale importanza, ben noto ma sempre irrisolto: la "fisica" impossibilità delle procure di far fronte al flusso delle notitiae criminis e la conseguente necessità di selezionarle a dispetto del principio di obbligatorietà dell'esercizio dell'azione penale. Il tutto aggravato da una debole propensione degli uffici verso modelli organizzativi di tipo innovativo.

Un argomento centrale in tutti gli studi sui sistemi giudiziari è rappresentato appunto dal carico di lavoro, analizzato distintamente nelle due grandi categorie della giustizia civile e della giustizia penale. ${ }^{7}$ E nell'ambito di questi due grandi settori della giurisprudenza si sono subiti gli effetti dell'irrazionale distribuzione delle risorse entro il gruppo di ricerca (dovuta a regole burocratiche), che ha posto severi limiti di metodo. Quanto alla giustizia civile, la ricerca si è quindi limitata a una minuziosa raccolta e interpretazione dei dati statistici sui flussi di litigiosità e sulla risposta del sistema alla domanda sociale di giustizia. Nel campo penalistico, la scelta dei ricercatori è stata quella di svolgere ricerche sul campo, qualitative e d'ambito limitato. In entrambi i settori, come già ricordato, sono state svolte osservazioni anche sugli strumenti di giustizia alternativa, che già all'inizio dello scorso decennio presentavano un certo grado di visibilità.

Il rapporto fra domanda e offerta di giustizia civile è un elemento essenziale per intendere il livello di salute di un intero sistema giudiziario. I flussi di litigiosità civile esprimono bisogni di soggetti privati individui e imprese - e, in grandissima maggioranza, coinvolgono valori economici, non importa se alti o modesti giacché il rilievo economico di una questione controversa deve essere valutato in relazione sia al reddito e al patrimonio, sia, e non meno, alla sensibilità degli interessati: negli studi sul cd. disputing è notorio che controversie di modesto valore economico spesso coinvolgono principi che le parti considerano non negoziabili, oltre a presentare problemi tecnico-giuridici di alta complessità. Nella ricerca, questo aspetto è stato anzitutto esaminato in uno studio di Stefania Pellegrini, dell'Università di Bologna, che ha colto l'occasione per aggiornare le osservazioni compiute in un suo libro del 1997 dedicato alla presentazione, elaborazione e analisi dei

7 L'indagine non ha potuto estendersi ad altre giurisdizioni, come quella amministrativa e quella contabile, che pure soffrono di gravi inefficienze e ritardi. 
dati statistici sulla litigiosità civile sull'arco di quasi un secolo. ${ }^{8}$ In quel volume, l'autrice aveva osservato che sull'efficienza del sistema e sulla propensione sociale ad avvalersi dei canali della giustizia interferiscono meno decisivamente le variabili esogene, quali l'andamento demografico, la situazione economica, gli indici di occupazione e simili, delle variabili endogene al sistema, cioè la sua organizzazione interna e i mutamenti normativi che periodicamente lo investono.

Nel suo contributo alla ricerca, ${ }^{9}$ la studiosa ha rilevato tendenze di analogo segno. Dal lato della domanda, i flussi di litigiosità hanno registrato un discreto aumento nei diversi gradi di giudizio, in particolare presso le corti d'appello, maggiormente onerate anche dalla riforma cd. del giudice unico, entrata in vigore alla fine degli anni ' 90 , che ha soppresso la figura del pretore e concentrato nei tribunali la gran parte del contenzioso di primo grado. Ha osservato l'autrice che sul numero di giudizi potrebbe influire anche il grande aumento del numero di avvocati, ma la correlazione fra tale dato e i procedimenti sopravvenuti in primo grado, da cui sono emerse percentuali medie irrisorie (da un massimo di 7,5 a un minimo di 1,2 nuovi procedimenti annui per professionista, a seconda delle sedi) ha messo in mostra soprattutto la crisi della classe forense, impegnata in una autentica "lotta per la sopravvivenza". Dal lato dell'offerta, la ricerca ha confermato il dato critico della grande lentezza dei giudizi, in primo come in secondo grado, accompagnato da una crescita del numero di giudizi esauriti senza sentenza, di solito corrispondenti ad accordi extragiudiziali raggiunti dalle parti. La crisi della giustizia, dunque, non si è attenuata, ma piuttosto aggravata, malgrado gli interventi legislativi intervenuti nel settore negli ultimi due decenni. Difficile, secondo Pellegrini, individuarne le cause, fra le tante possibili: incompletezza degli organici, soprattutto nei ruoli ausiliari, deficienze infrastrutturali anche nella gestione dei singoli procedimenti, difforme dislocazione del personale, variabile e a volte insufficiente impegno da parte dei giudici, sovraccarico del lavoro penale che rischia di soffocare quello civile.

$\mathrm{Nel}$ complesso, questo studio descrive una situazione che induce

8 Pellegrini S. La litigiosità in Italia. Un'analisi sociologico-giuridica. Giuffrè, Milano, 1997.

9 Pellegrini S. Il contenzioso giudiziario in Italia tra mutamento e riforme. Giuffrè, Milano, 2008. 
a cercare vie alternative a quelle tradizionali, interne o esterne al sistema. Fra le prime, rileva in primo luogo la necessità di procedere urgentemente a una ridefinizione della geografia giudiziaria secondo criteri organizzativi razionali: argomento ricorrente ma, come noto, delicatissimo per le implicazioni politico-territoriali che spesso paralizzano ogni tentativo, appunto, di razionalizzazione. In secondo luogo, rileva l'innovazioni tecnologica, in particolare per quel che concerne la cd. $e$ justice. Sull'informatizzazione degli uffici e delle procedure, la studiosa bolognese ha manifestato fiducia in teoria ma sfiducia in pratica, osservando che su questa strada sono disseminati ostacoli burocratici e culturali. E questa ambiguità di fondo emerge anche da alcuni studi, nati originariamente al di fuori del progetto di ricerca, che però li ha acquisiti in itinere per il loro oggettivo interesse. In questi lavori, ${ }^{10} \mathrm{i}$ ricercatori dell'Istituto di ricerca sui sistemi giudiziari del Consiglio Nazionale delle Ricerche, all'epoca diretto da Giuseppe Di Federico, hanno parlato di successi e di delusioni, osservando (anche comparativamente con altre esperienze europee) che accanto a "notevoli passi avanti" che hanno dotato l'Italia di "una buona piattaforma tecnologica" nella giustizia, ${ }^{11}$ si devono notare anche difficoltà di applicazione nel cd. processo telematico ${ }^{12} \mathrm{e}$, in genere, una visibile sproporzione fra le iniziative attuate e gli alti costi relativi, da un lato, e dall'altro lato i risultati acquisiti, ostacolati anche "da un apparato normativo assolutamente sovradimensionato rispetto alle poche applicazioni realmente funzionanti a livello nazionale". ${ }^{13}$

Fra le alternative "esterne" al sistema della giustizia civile, è emerso in primo piano nel corso della ricerca l'argomento dei cd. metodi di ADR (Alternative Dispute Resolution), dibattuto in ogni sede negli ultimi decenni e oggetto anche di impegni politici assunti, per esempio, nell'ambito europeo.

10 Carnevali D., Contini F. \& Fabri M. (a cura di). Tecnologie per la giustizia. I successi e le false promesse dell'e-justice. Prefazione di Di Federico G., Giuffrè, Milano, 2006.

11 Fabri M. Organizzazione giudiziaria e tecnologie, in Tecnologie per la giustizia, cit., p. 38.

12 Contini F. L'infrastruttura dell'informazione, in Tecnologie per la giustizia, cit., p. es. p. 80.

13 D. Carnevali, L'Italia nel tunnel dell'e-justice, in Tecnologie per la giustizia, cit., p. 132. 
Il primo volume uscito nell'ambito della ricerca, ${ }^{14}$ frutto di una tesi di dottorato venuto ad arricchirla nel corso del suo svolgimento, ha contribuito a tracciare un quadro generale del problema, avvalendosi di un fine impianto teorico e attingendo a una ricca letteratura soprattutto americana, giacché negli Stati Uniti la questione delle ADR e della giustizia cd. informale si è posta già negli anni '70, in forte anticipo rispetto all'Europa. L'autore, Ivan Pupolizio, ha distinto fra le diverse esperienze di giustizia alternativa e individuato in particolare tre modelli teorici di mediazione ("negoziale", "comunitaria" e "terapeutica"), ha successivamente concentrato l'attenzione su una specifica esperienza americana (il San Francisco Community Boards Program), per poi compiere osservazioni sulla (modesta) attività di alcuni centri di mediazione sociale in Italia, operanti all'inizio dello scorso decennio. Lo studioso ha concluso mettendo in rilievo, grazie anche ad osservazioni qualitative, la possibilità che i procedimenti mediatori riescano nell'intento di risolvere i conflitti mantenendo, o ricostruendo, il sottostante rapporto fra le parti. Ha anche osservato, per contro, che la conflittualità sociale cerca soluzione non solo nella pacificazione, ma anche in una decisione dirimente su ragioni e torti: e qui il ruolo del giudice non è surrogabile.

Anche Stefania Pellegrini ha gettato lo sguardo sulle ADR nella seconda parte del libro appena citato, raccogliendo dati sulle (poche) istituzioni svolgenti attività di mediazione, arbitrato e conciliazione, quali le Camere di Commercio e i CORECOM. La realtà che ne è uscita è apparsa modesta, anche se non deludente, soprattutto per i tempi brevi di smaltimento delle pratiche. In secondo luogo, la studiosa ha discusso il caso specifico dei conflitti coniugali, che notoriamente si svolgono e si esauriscono, di fatto, fuori dei tribunali, ma ne aggravano il lavoro per la perdurante necessità dell'intervento giudiziale confermativo di accordi già raggiunti stragiudizialmente, spesso grazie all'attività mediatrice degli avvocati delle parti. Di questa litigiosità fittizia ha osservato - la giustizia formale dovrebbe liberarsi, mentre dovrebbe essere incrementata l'attività di centri di mediazione familiare che possano, fra l'altro, intervenire nel conflitto secondo tempi più adeguati

14 Pupolizio I. Una comunità all'ombra del diritto. La mediazione sociale e la giustizia informale nel modello statunitense e nell'esperienza italiana. Giuffrè, Milano, 2005. 
alle sue tappe effettive, legate all'esperienza psicologica e affettiva del conflitto stesso.

Nel complesso, la ricerca ha rivelato che il tema della mediazione (e di esperienze consimili) è importante e promettente, ma delicato e complesso, trattabile solo alla luce di conoscenze precise dei bisogni della gente e dei corrispondenti interessi. Per questo è apparsa stupefacente la riforma che nel 2010 ha affrontato l'argomento imponendo per certe materie (fra cui quella delicatissima della responsabilità del medico) la mediazione come esperimento preliminare all'instaurazione di una lite per le vie ordinarie. Gli effetti di questa legge, operativa dall'aprile 2011, sono ancora da studiare, ma la rozzezza e l'improvvisazione con cui è stata concepita non lasciano ben sperare.

A cavallo, per dir così, fra rimedi interni ed esterni alla crisi della giustizia, si colloca infine l'esperienza dei giudici di pace, istituiti nel 1991 - in sostituzione del vecchio giudice conciliatore - come giudici onorari appartenenti all'ordine giudiziario ma immaginati dal legislatore sia come bacino scolmatore di una giustizia onerata da troppe piccole controversie, sia come luogo di "socializzazione" dei conflitti, di incanalamento degli stessi lungo percorsi informali non molto dissimili dai metodi di ADR appena ricordati. Su questo argomento, si è svolto nella ricerca uno studio specifico, purtroppo stroncato nella fase conclusiva dalla grave infermità che avrebbe portato nel 2007 alla prematura scomparsa dell'autore, Odillo Vidoni Guidoni, ricercatore dell'Università di Torino. A dispetto di ciò, il volumetto che ne è scaturito $^{15}$ è un lavoro esemplare. Fondando le sue ipotesi su un fine apparato teorico e traendo argomenti da osservazioni empiriche sull'attività del giudice di pace, l'autore ha riscontrato in questa magistratura onoraria elevati indici di efficienza e capacità di operare in senso deflattivo sul fronte della litigiosità, ma al tempo stesso un sostanziale mutamento avvenuto nel suo ruolo istituzionale rispetto alle originali intenzioni del legislatore. Il giudice di pace infatti si è progressivamente distanziato dalla primitiva immagine, in certo modo alternativa e "sociale" rispetto al giudice ordinario, per acquisire tratti sempre più vicini a quelli del giudice professionale, anche per l'accesso sempre più massiccio tra le sue file di giovani avvocati privi di un lavoro sicuro e l'esten-

15 Vidoni Guidoni O. Quale giustizia per il giudice di pace? Nascita e consolidamento di una magistratura onoraria. Giuffrè, Milano, 2006. 
sione progressiva delle competenze, che ora abbracciano dispute civili di rimarchevole valore e perfino processi penali. Le conclusioni di questa ricerca - lo dico incidentalmente - hanno confermato una tendenza già osservata in altri contesti e paesi dagli studiosi della litigiosità e della giustizia cd. informale.

Il campo della giustizia penale, benché parzialmente sacrificato come già ricordato - dalla ineguale distribuzione delle risorse fra i gruppi di ricerca, è stato oggetto di due studi di sicuro interesse, l'uno qualitativo, l'altro quantitativo.

Il primo studio, svolto presso l'Università di Torino da Claudio Sarzotti con l'ausilio di Cecilia Blengino e Giovanni Torrente, ha affrontato il tema dell'esercizio dell'azione penale in rapporto all'organizzazione delle Procure della Repubblica. ${ }^{16} \mathrm{Si}$ tratta, come noto, di uno dei problemi più acuti dell'intero sistema della giustizia in Italia, ben sintetizzato dalle parole di un magistrato, poste dagli autori come epigrafe del loro volume: "La delinquenza è talmente aumentata negli ultimi anni che il processo, così com'è strutturato oggi, è un lusso che la società non si può più permettere”. Oberato dagli affari penali, già incombenti in passato ${ }^{17}$ ma sempre crescenti nei numeri e sempre più complessi anche dal punto di vista delle incombenze formali, l'intero sistema di giustizia è ormai al collasso, con conseguenze perniciose. Se la probabilità statistica di incorrere nella repressione penale è modesta, il reato diventa un rischio che per alcuni può valer la pena di correre.

La crisi della giustizia penale, che si riverbera su tutto il sistema, nasce sin dall'origine, cioè dal flusso di notizie di reato che ogni giorno perviene alle Procure e che queste, come già detto, non possono smaltire a dispetto del principio di obbligatorietà dell'azione penale, che pure le vincola. Pertanto i processi che emergono dal mucchio sono frutto di una selezione, inevitabile ma paradossale: se avviene in modo ponderato, badando a priorità ritenute oggettive come la gravità del reato, il rischio sociale che vi è connesso o la complessità delle procedure, ne possono derivare serie conseguenze a carico dell'ufficio; se, per contro, avviene in modo casuale, è possibile che proprio i reati più gravi

16 Sarzotti C. Processi di selezione del crimine. Procure della Repubblica e organizzazione giudiziaria. Con saggi di Blengino C., Torrente G. e prefazione di Maddalena M. Giuffrè, Milano, 2007.

17 Resta E. Conflitti sociali e giustizia. De Donato, Bari, 1977. 
rimangano impuniti. È precisamente su questo tema, cioè sul cammino percorso dalle notitiae criminis, che si è svolta la ricerca degli studiosi torinesi, condotta presso due Procure della Repubblica, Torino e Bari, differenziate proprio su questo punto nevralgico, giacché la prima ha seguito per anni dei criteri razionali di selettività perseguendo finalità di efficienza e di produttività, mentre la seconda ha preferito "orientare i propri processi organizzativi ad un rispetto formale dell'obbligatorietà dell'azione penale", con relativo sacrificio dell'efficienza e della produttività, seppur nel quadro di una politica di serio contrasto alla criminalità organizzata. ${ }^{18}$ Due modelli - definiti rispettivamente "efficientista" e "fatalista" 19 fra i quali è difficile scegliere. L'uno, meno rispettoso del principio di obbligatorietà dell'azione penale, si mostra capace di raggiungere finalità immediate anche sul piano delle formalità processuali che spesso inceppano i procedimenti, ma altrettanto spesso riflettono diritti soggettivi non sacrificabili degli imputati. L'altro, ineccepibile sul piano formale, non può raggiungere i suoi scopi istituzionali se non in modo imprevedibile. Nell'impossibilità di risolvere il dilemma, è probabile che il sistema, per dir così, scelga da sé o, per meglio dire, venga piegato da scelte preponderanti del legislatore che negli ultimi anni è sembrato inclinare pericolosamente verso un sistema di repressione selettivo all'origine, attraverso la depenalizzazione totale o parziale di alcuni reati "di colletti bianchi" - esemplare il falso in bilancio - e, per contro, l'apertura di una "via italiana verso quel paradigma del 'diritto penale del nemico", ${ }^{20}$ rivolto soprattutto contro le fasce deboli e marginali (piccoli spacciatori, immigrati clandestini e simili) e teorizzato da alcune dottrine penalistiche non solo italiane, influenzate dal neofunzionalismo sociologico tedesco.

Lo studio quantitativo ha affrontato, in modo quasi pionieristico rispetto all'esperienza italiana, la mediazione penale, con cui si cerca al tempo stesso di deflazionare la giustizia penale e di renderla meno dura

18 Blengino C. Esercizio dell'azione penale e processi organizzativi: la selezione del crimine come output della Procura. In Sarzotti C. et al. Processi di selezione del crimine. Cit., pp. 222-3.

19 Torrente G. Le storie organizzative di due Procure della Repubblica tra obbligatorietà dell'azione penale e selezione del crimine. In Sarzotti C. et al. Processi di selezione del crimine. Cit., pp. 227 ss.

20 Sarzotti C. Le Procure della Repubblica come attori del campo penale. In Sarzotti C. et al. Processi di selezione del crimine. Cit., p. 116. 
e cogente nei confronti di alcune fasce di persone. Fra queste, rileva soprattutto quella minorile, su cui si sono appuntati i ricercatori, Laura Vaira, sociologa del diritto e operatrice del settore, e Alberto Nosenzo, giurista e attualmente magistrato del Tribunale di Milano. ${ }^{21}$ La loro ricerca, inquadrato il tema nel contesto della giustizia "riparativa", volta a ripristinare il rapporto sociale leso da un comportamento deviante, ha preso in esame otto uffici di mediazione operanti al tempo, relativamente al periodo 1995-2004, e ne ha registrato l'attività a partire dal flusso dei fascicoli pervenuti, proseguendo per l'individuazione dei criteri di selezione adottati e delle norme applicate, per finire con la registrazione degli esiti della procedura, positivi e negativi. Dai dati è emersa una realtà modesta dal punto di vista numerico anche se, caso per caso, coronata da discreto successo, visto che le percentuali andavano da un minimo del $35 \%$ nell'Ufficio di Torino a un massimo del $73 \%$ nell'Ufficio di Catanzaro e il mancato successo dipendeva più da ragioni obiettive che dal difetto di volontà delle parti contrapposte. $\mathrm{Ma}$ si trattava comunque - si ripete - di numeri modesti, a fronte dei quali era logico concludere che, quanto meno dal punto di vista dell'effetto deflattivo, l'istituto della mediazione appariva inadeguato, anche perché implicante un notevole dispendio di forze e di attività. Del resto, è spontaneo osservare che se la mediazione deve avere una funzione ricostruttiva di rapporti sociali interrotti, essa si può esplicare solo attraverso una lunga e difficile opera anche psicologica, chiaramente incompatibile con le necessità di accelerazione temporale che stanno a base delle strategie deflattive nel processo.

La realtà, emersa da entrambi questi studi, riconduce sempre al punto di partenza, già osservato nel campo della giustizia civile: il sistema, soffocato da un flusso eccessivo di input, non riesce "fisicamente" ad assorbirlo in tempi adeguati. Le alternative sinora individuate, ispirate fra l'altro a finalità diverse da quella della pura efficienza, non sembrano in grado in se stesse di risolvere il problema, anche perché la giustizia ordinaria persegue fini istituzionali ai quali lo Stato non può abdicare.

Messa in luce questa realtà, va pur detto che essa non si presenta con gli stessi caratteri in tutte le parti d'Italia. Uno studio economico,

21 Vaira L., Nosenzo A. Uffici di mediazione penale e magistratura minorile in Italia. In Cominelli L. (a cura di). Temi e problemi della giustizia. Giuffrè, Milano, 2007, pp. 15 ss. 
svolto da Riccardo Marselli e Marco Vannini, rispettivamente dell'Università Parthenope di Napoli e dell'Università di Sassari, si è rivolto precisamente a verificare gli standard di efficienza dei diversi uffici giudiziari della penisola nel rispondere alla domanda di giustizia. ${ }^{22}$ Inquadrato il lavoro come studio dell'efficienza nell'ambito delle frontiere di produzione, e fatta applicazione del metodo DEA (Data Envelopment Analysis), gli autori hanno misurato i livelli di efficienza di tutti i distretti di Corte d'Appello italiani sulla base di alcune variabili: numero di magistrati operativi, pendenze, procedimenti sopravvenuti, procedimenti definiti, sia nel civile sia nel penale. Il calcolo ha permesso di individuare 12 distretti efficienti e 17 inefficienti su un totale di 29, con punte massime di efficienza a Bolzano, Brescia e L'Aquila, e punte massime di inefficienza in alcune sedi particolarmente grandi, come Genova, Milano, Napoli, Roma e Torino. L'inefficienza - si è osservato - dipende soprattutto da "un rilevante carico di procedimenti pendenti, soprattutto in materia penale" e va valutata anche nella prospettiva delle economie di scala per alcuni distretti troppo grandi o troppo piccoli rispetto al carico di lavoro generale: osservazione, quest'ultima, che depone per l'ennesima volta a favore di una sostanziale revisione della geografia giudiziaria italiana.

Esaurita la descrizione dei risultati concernenti il sistema della giustizia nella sua struttura e nelle sue funzioni, affronterò ora quelli concernenti il rapporto fra il sistema stesso e altri sistemi d'azione sociale, sempre ribadendo che la distinzione fra sistemi e i relativi confini sono effetto di scelte operate essenzialmente a fini analitici, ma in certa misura arbitrarie se si considera che l'apertura sistemica, salvo eccezioni, è un elemento comune anche presso la maggioranza degli scienziati orientati in senso sistemico.

$\mathrm{Su}$ questo fronte, la ricerca ha preso in esame quattro diversi ambiti di comunicazione inter-sistemica, esaminando le relazioni del sistema giuridico, rispettivamente, con la famiglia, la politica, il contesto europeo e l'avvocatura. Su ciascun argomento sono apparsi studi monografici che non intendono esaurire il campo delle osservazioni possibili, giacché il diritto, come ovvio, si occupa o può occuparsi di

22 Marselli R., Vannini M. L'efficienza tecnica dei Distretti di Corte d'Appello italiani. Aspetti metodologici, benchmarking e arretrato smaltibile. In Cominelli L. (a cura di). Temi e problemi della giustizia. Giuffrè, Milano, 2007, pp. 75 ss. 
qualsiasi ambito d'azione umana, ma offrono spunti non secondari per l'approfondimento di problemi spesso acuti e pur tuttavia trattati superficialmente nelle pubbliche discussioni.

In tema di famiglia, un gruppo di ricerca coordinato da Paola Ronfani dell'Università di Milano e composto altresì da Flavio Ceravolo e Noemi Podestà dell'Università di Torino, Anna Rosa Favretto dell'Università del Piemonte Orientale e Valerio Pocar dell'Università di Milano-Bicocca, ha incentrato l'attenzione su un elemento di intuibile importanza, l'orientamento dei giudici rispetto ai valori socio-familiari. A tal uopo, i ricercatori hanno sottoposto un questionario a un campione rappresentativo, i magistrati ordinari e onorari impegnati negli affari familiari, chiedendo loro di pronunciarsi su una serie di quesiti cruciali nel quadro di quella visione "mobile" che caratterizza la famiglia contemporanea in una società pluralista: che cosa s'intende per famiglia, quali le cause della sua crisi, la famiglia di fatto, le unioni omosessuali, i rapporti tra figli e genitori, biologici e "sociali", la fecondazione eterologa, l'affidamento congiunto, i ruoli istituzionali connessi al conflitto familiare. ${ }^{23}$ Le risposte, classificate secondo uno scalogramma basato su due coppie di variabili - familismo-legalismo e tradizione-innovazione hanno lasciato i ricercatori in qualche modo perplessi, giacché non hanno rivelato tendenze univoche e neppure variazioni che potessero trovare adeguata spiegazione in riferimento ai modelli interpretativi adottati (peraltro comuni negli studi di sociologia della famiglia e del relativo diritto), tanto che Paola Ronfani, nelle sue conclusioni, ha ritenuto di metterli in discussione quali "strumenti euristici" e osservato che gli operatori intervistati sembrano "orientarsi verso modelli di giustizia familiare compositi, spuri e di tipo sincretico". ${ }^{24}$ In proposito, si può osservare che soprattutto in periodi di accelerato mutamento sociale non è da attendersi che magistrati di diversa età, genere, provenienza e anche ruolo professino valori comuni. $\mathrm{Ma}$, pur dando atto che le disaggregazioni non hanno messo in luce variazioni significative, si deve anche rilevare che su alcuni punti si sono riscontrati atteggiamenti maggioritari interes-

23 Ronfani P. (a cura di). Quale giustizia per le famiglie? Gli orientamenti della cultura giuridica nella società che cambia. Con contributi di Ceravolo F., Favretto A.R., Pocar V., Podestà N. \& Ronfani P. Giuffrè, Milano, 2006.

24 Ronfani P. Le incertezze della giustizia familiare. In Ronfani P. (a cura di), Quale giustizia per le famiglie? Cit., p. 169. 
santi, per esempio in tema di famiglia di fatto, fecondazione eterologa e patti di unione civile anche per coppie omosessuali. ${ }^{25}$ Dalle interviste è emerso anche, con prepotenza, il tema del minore come soggetto di diritto. L'istituzione gli deve "protezione" o piuttosto riconoscimento di diritti fondamentali? Dietro questo dilemma, messo in luce da Valerio Pocar, ${ }^{26}$ vi è una diversa concezione del ruolo del giudice minorile, su cui la discussione prosegue.

Il tema, delicatissimo da almeno trent'anni, del rapporto fra giustizia e politica è stato esaminato da Morris L. Ghezzi e Marco A. Quiroz Vitale, dell'Università di Milano, attraverso una doppia indagine, quantitativa e qualitativa, vertente sull'immagine pubblica della magistratura italiana. ${ }^{27}$

La prima indagine è consistita in due rilevazioni svolte nella primavera e nell'autunno 2003 con la tecnologia Eurotop dall'Istituto di ricerca CIRM di Milano sotto la direzione dello stesso Ghezzi. Ai due diversi campioni di intervistati sono state rivolte, mediante un articolato questionario, numerose domande incentrate sul ruolo, l'efficienza e la credibilità dell'amministrazione della giustizia. Le risposte ottenute sono state altrettanto articolate e di grande interesse. Sinteticamente, esse hanno indicato una sostanziale sfiducia della popolazione verso il sistema della giustizia, sia per i tempi lunghissimi con cui lavora, ritenuti il suo difetto fondamentale, sia per la sua attitudine, storica, a perpetuare le disuguaglianze sociali, ma al tempo stesso un'opinione positiva sui giudici, ritenuti in genere preparati e affidabili. Questa divaricazione è indice di discernimento critico, confermato dalle opinioni su argomenti specifici. Per esempio, relativamente al ruolo e alle funzioni del pubblico ministero, la maggioranza si è espressa contro la separazione delle carriere; ha ritenuto che il pubblico ministero vada scelto per concorso, non eletto dai cittadini; ha ritenuto che il pubblico ministero selezioni i reati da perseguire secondo criteri di preferenza (in particolare per la corruzione politica) e rivendicato maggior protezione

25 V. la presentazione critica dei dati in Favretto A.R., Ceravolo F. Famiglia e giustizia: gli orientamenti della cultura giuridica. In Ronfani P. (a cura di). Quale giustizia per le famiglie? Cit., pp. 91 ss.

26 Pocar V. La giustizia familiare. In Ronfani P. (a cura di). Quale giustizia per le famiglie? Cit., p. 71 ss.

27 Ghezzi M.L., Quiroz Vitale M.A. L'immagine pubblica della magistratura italiana, Giuffrè, Milano, 2006. 
contro la microcriminalità. Disaggregando le risposte, è emerso prepotentemente il peso della variabile "politica". Fra rispondenti di centrodestra, centro e centro-sinistra si sono manifestate variazioni d'opinione talmente vistose - col centro-destra in chiarissima evidenza nel criticare l'operato della magistratura - da chiedersi quanto abbiano influito su di esse le campagne mediatiche iniziate con la vicenda di Tangentopoli e proseguite per anni. Lo stesso Ghezzi ha rilevato sin dall'introduzione al volume questa dipendenza delle opinioni dai media. ${ }^{28}$ Ferme restando queste considerazioni, va certamente osservato che, al di là delle singole fattispecie prospettate agli intervistati, questi hanno espresso, come già detto, una fondamentale sfiducia nell'istituzione giudiziaria, segnalando che la sua crisi è socialmente percepita quasi come un dato di fatto. Da qui sono possibili varie conseguenze a livello prescrittivo. La maggioranza dei rispondenti sembra suggerire una soluzione riformistica, di correzione interna del sistema. Soluzione che, a mio avviso, parte dalla constatazione che in materia di diritti e doveri tocca allo Stato, quale organizzazione politica della cittadinanza, dire la parola decisiva attraverso giudici indipendenti. Ghezzi, dal canto suo, dichiara di non invadere il terreno prescrittivo in quanto estraneo alla natura empirica della sociologia del diritto, e tuttavia, insistendo su un tema a lui caro e successivamente ribadito in altre pubblicazioni, ${ }^{29}$ parla di "nichilismo giuridico", o meglio di "nihilismo" (ispirandosi soprattutto alla sociologia giuridica di Theodor Geiger), e mette in discussione la sovranità dello Stato, il principio di maggioranza e conseguentemente lo stesso diritto-dovere dello Stato di far giustizia in una società "liquida", ${ }^{30}$ contrapponendovi la figura di un giudice "quivis de populo" sin dall'introduzione al volume.

I risultati dell'indagine quantitativa sono stati successivamente presentati e discussi in quattro focus groups, composti da avvocati del Foro

28 Ghezzi M.L. Il giudice come "quivis de populo". Intr. a Ghezzi M.L., Quiroz Vitale M.A. L'immagine pubblica della magistratura italiana. Cit., p. 9.

29 V. p. es. Ghezzi M.L. La scienza del dubbio. Volti e temi di sociologia del diritto. Mimesis, Milano, 2009.

30 Ghezzi M.L. La percezione dell'amministrazione della giustizia tra gli italiani: interpretazioni ed ipotesi intorno ai dati quantitativi raccolti. In Ghezzi M.L., Quiroz Vitale M.A. L'immagine pubblica della magistratura italiana. Cit., p. 17 ss. È esplicito il riferimento dell'autore (p. es. p. 5) alla teoria di Zygmunt Bauman, esposta nel classico Vita liquida [2005], tr. it., Laterza, Roma-Bari, 2006. 
di Milano, giovani universitari, operatori sociali e pubblici impiegati e coordinati da Marco A. Quiroz Vitale. Lo stesso Quiroz Vitale ha altresì svolto interviste strutturate mediante questionari inviati per posta elettronica a testimoni privilegiati (magistrati, avvocati, politici, giornalisti e imprenditori). Tutto ciò ha dato linfa alla seconda parte dell'indagine, qualitativa, ${ }^{31}$ in cui il giudizio sostanzialmente negativo verso la giustizia italiana, per il suo carattere inegualitario, la sua lentezza endemica, l'influenzabilità dei giudici, è stato confermato, seppur con toni non enfatici, sia nelle opinioni degli intervistati, sia nel corso delle discussioni coordinate di gruppo. Ispirandosi a fonti in parte simili in parte diverse da quelle di Ghezzi - comune il riferimento a Zygmunt Bauman, distinto il richiamo al grande sociologo italo-argentino Gino Germani e, alla fine, perfino a don Lorenzo Milani - Quiroz ne ha condiviso in parte, più timidamente, anche le conclusioni, esprimendosi anche lui per un giudice "quivis del populo", vicino alla società e dotato di discrezionalità: in tali casi "la scelta inevitabile tra differenti corsi d'azione o inazione [...] è meno problematica perché aver fiducia nel discernimento del decisore o del giudice è, in sostanza, come aver fiducia di se stessi" ${ }^{32}$

Il tema trattato da Ghezzi e Quiroz Vitale è destinato a ulteriori discussioni, benché sia da sperare che in una nuova fase della politica italiana il conflitto che da trent'anni oppone la classe politica e la magistratura, se non venga a cessare, almeno perda di asprezza.

A nuove discussioni è pure destinato - anche qui, si spera, in un clima più propizio di quello corrente - il tema del rapporto fra giustizia italiana e giustizia europea (in senso lato, comprendente sia l'Unione Europea, sia il Consiglio d'Europa). Su questo argomento, la ricerca ha acquisito tre contributi di rilievo, rispettivamente dedicati al "dialogo fra giudici" italiani e comunitari, alla giurisprudenza della Corte di Strasburgo sul cd. délai raisonnable nei giudizi e alla dimensione continentale dell'avvocatura: tema, quest'ultimo, che ha fatto da ponte ideale con la questione del rapporto fra magistratura e avvocatura, storicamente delicato e pur esso molto caldo negli ultimi anni.

31 Quiroz Vitale M.A. Il ruolo del magistrato e l'immagine nella ricerca qualitativa. In Ghezzi M.L., Quiroz Vitale M.A. L'immagine pubblica della magistratura italiana. Cit., pp. 239 ss.

32 Quiroz Vitale M.A. Il ruolo del magistrato e l'immagine nella ricerca qualitativa. Cit., p. 397 (corsivo nel testo). 
Il dialogo fra giudici, argomento centrale nell'evoluzione del diritto contemporaneo caratterizzato da una molteplicità di fonti su scala sovranazionale, è stato affrontato da M. Cristina Reale, docente dell'Università dell'Insubria e responsabile del progetto specifico, e Marco Borraccetti, al tempo dottorando e ora docente dell'Università di Bologna, sotto un profilo specifico, ma di particolare significato per il sistema comunitario: quello dei rinvii pregiudiziali alla Corte di Giustizia delle Comunità Europee. ${ }^{33}$ Questo istituto, in senso (molto) lato paragonabile al rinvio alle Corti costituzionali nazionali, rappresenta uno specchio molto significativo sia delle eventuali discrepanze fra diritto nazionale e diritto comunitario, sia del livello di conoscenza che giudici e giuristi nazionali posseggono del diritto comunitario. La ricerca, inquadrata da Marco Borraccetti sotto il profilo giuridico-formale, è stata svolta sul piano empirico da $\mathrm{M}$. Cristina Reale con un metodo già da lei utilizzato efficacemente in un precedente lavoro, ${ }^{34}$ attraverso un'analisi del contenuto di tutti i rinvii pregiudiziali disposti dai giudici italiani sino a tutto il 2007, a partire dal 1964: con un caso, Costa c. Enel, che nacque in Italia come un escamotage (fallito) volto a paralizzare sul nascere la legge di nazionalizzazione delle imprese elettrice, ma determinò una decisione della Corte di Giustizia CEE fra le più decisive per la costruzione dell'ordinamento comunitario. Questo promettente avvio, dovuto all'interessamento personale del capo dell'Ufficio di conciliazione di Milano, non sembra aver avuto adeguati sviluppi nei decenni successivi. I 939 rinvii pregiudiziali provenuti a Lussemburgo da parte italiana sull'arco di più quarant'anni sono infatti relativamente pochi se rapportati alle molteplici incompatibilità riscontrabili nella legge italiana rispetto alla normativa comunitaria. $\mathrm{Ma}$ a parte questo rilievo - riproponibile anche per altri paesi membri, peraltro con eccezioni (l'Austria, aderente alla CEE nel 1995, ha proposto in poco più di un decennio ben 308 rinvii, il $25,9 \%$ del totale assoluto) - ciò che più colpisce delle rilevazioni effettuale è un alto indice di casualità, sia nella giurisdizione che ha disposto i rinvii, sia nelle materie interessate, sia nel foro di provenienza. Fra le giurisdizioni pre-

33 Reale M.C., Borraccetti M. Da giudice a giudice. Il dialogo tra giudice italiano e Corte di giustizia delle Comunità europee. Giuffrè, Milano, 2008.

34 Reale M.C. Il Tribunale di primo grado e la litigiosità comunitaria. Un'indagine sociologico-giuridica, Giuffrè, Milano, 2000. 
valgono naturalmente $\mathrm{i}$ giudici di primo grado (preture, tribunali, TAR), al cui proposito si dovrebbe osservare che la Corte di Cassazione, suprema regolatrice del diritto, avrebbe potuto essere più attiva, gravando su di essa un obbligo preciso di disporre il rinvio. Fra le materie, non sorprende (e il dato è interessante) che prevalgano le questioni afferenti all'agricoltura, argomento dolente della politica comunitaria non solo italiana. Tra i fori di provenienza è apparsa sorprendente la prevalenza massiccia dei rinvii disposti a Genova: ma il dato, commentato pubblicamente nei convegni in cui l'indagine generale è stata discussa, ha trovato una semplice spiegazione nel fatto che la fonte primigenia fosse quasi sempre lo stesso studio legale. Questo elemento, apparentemente casuale, è in realtà sintomatico di una situazione generale. La conoscenza del diritto comunitario in Italia, da parte dei giudici come degli avvocati, è in effetti casuale e distribuita a pelle di leopardo. A ciò, si può aggiungere la resistenza naturale dei giudici e degli stessi avvocati a distanziarsi dalla legge italiana. Ed è sempre il dato culturale che, a quanto sembra, fa difetto.

Bruno Nascimbene e Cecilia Sanna, internazionalisti dell'Università di Milano, hanno affrontato uno dei punti più dolenti dell'intera giurisdizione italiana, cioè le sentenze della Corte europea dei diritti dell'uomo sui casi di "ritardo irragionevole" riguardanti la nostra giustizia. ${ }^{35} \mathrm{Il}$ "caso Italia" è purtroppo noto, da sempre, e altrettanto imbarazzante. Le sentenze di condanna per irragionevole ritardo nell'emettere decisioni giudiziarie riguardano per una larga maggioranza relativa (in alcuni anni prossima alla maggioranza assoluta) precisamente il nostro paese: il quale, per perfido paradosso, è stato persino condannato due volte per la stessa procedura! ${ }^{36}$ Il flusso si è attenuato dopo il 2001, anno di emanazione della cd. Legge Pinto che peraltro, ancorché doverosa dal punto di vista formale, ha aggravato il problema anziché risolverlo, imponendo alle parti danneggiate dal ritardo irragionevole l'onere di chiederne in giudizio la riparazione al patrio governo come condizione per poter adire la Corte europea. I ritardi quindi si

35 Nascimbene B., Sanna C. L'eccessiva durata dei processi in Italia e le conseguenze a livello europeo. In Cominelli L. (a cura di). Temi e problemi della giustizia. Cit., pp.169 ss.

36 Nascimbene B., Sanna C. L'eccessiva durata dei processi in Italia e le conseguenze a livello europeo. Cit. p. 184. 
sono ulteriormente accumulati, come Nascimbene e Sanna puntualmente hanno registrato al termine del loro contributo.

Gli stessi autori hanno ulteriormente contribuito alla ricerca come già detto - attraverso uno studio sulla circolazione degli avvocati in Italia. ${ }^{37} \mathrm{Al}$ tempo dell'indagine questo fenomeno aveva dimensioni numeriche modeste, sebbene qualitativamente non irrilevanti considerando la partecipazione degli avvocati stranieri nei grandi studi associati. L'Italia, in certo modo, è dunque apparsa anche su questo punto alquanto isolata, malgrado le (relative) aperture giurisprudenziali. Fra le cause, gli autori hanno segnalato gli attriti derivanti dalla resistenza corporativa degli Ordini forensi italiani e il sistema delle tariffe. Resta a vedere se il recente cambiamento di queste ultime, che peraltro ha posto più problemi, tuttora aperti, di quanti non ne abbia risolti, varrà ad aprire le porte del paese alla libera circolazione di questi professionisti. Nel frattempo, tuttavia, la chiusura nazionalistica si manifesta ovunque in Europa a livelli ben più generali di quello di una singola professione libera.

Infine, Vittorio Olgiati e Francesca Tacchi si sono impegnati nello studio in chiave storico-interpretativa dell'avvocatura nei suoi rapporti con il sistema della giustizia, partendo dal presupposto - in sé corretto, sebbene espresso in forme alquanto radicali - della sostanziale estraneità del mondo forense rispetto a quel sistema. Questo contributo, ultimato nella ricostruzione storica di Francesca Tacchi e giunto allo stato di avanzata elaborazione nell'interpretazione sociologica di Vittorio Olgiati, non è arrivato alla definitiva conclusione. Dalle relazioni degli autori ai convegni e dalle bozze circolate in vista della pubblicazione, si possono comunque trarre alcuni dati di sintesi.

L'attento studio storico di Francesca Tacchi, dell'Università di Firenze, ha descritto il difficile rapporto fra le due grandi componenti della vita giudiziaria a partire dall'Unità d'Italia e lungo tutte le diverse fasi della storia nazionale fino agli anni ' 70 . Il panorama da lei descritto è quello di una permanente conflittualità, ora latente ora manifesta, che si è dipanata nei decenni senza trovare durature soluzioni. La studiosa ha incentrato l'attenzione sul passaggio dal fascismo - quando a

37 Nascimbene B., Sanna C. La circolazione degli avvocati in Italia tra norme comunitarie e norme interne. In Cominelli L. (a cura di). Temi e problemi della giustizia. Cit., pp. 115 ss. 
una sostanziale e sostanziosa fascistizzazione della magistratura fece riscontro un esautoramento della funzione dell'avvocatura (sancita da una legge professionale ancora in vigore) - alla Repubblica. Il suo momento fondante, la Costituzione, pur nella sua tardiva attuazione, ha sancito l'esistenza di un potere giudiziario a fronte, però, di un'avvocatura frammentata ma capace di ergersi in varie occasioni, specialmente negli anni '60 e '70, a difesa dell'ordine democratico nei momenti più difficili della vita nazionale: non senza, peraltro, una crescente sfiducia rispetto ad una amministrazione "dirigistica" della giustizia, che invita alla "fuga" verso altre forme di tutela dei diritti.

Passando a un livello d'analisi sociologico-giuridico, Vittorio Olgiati (al tempo docente dell'Università di Urbino, oggi professore dell'Università di Macerata), ha rappresentato l'avvocatura nel quadro della "ambivalenza delle dinamiche tecnico-giuridiche, ideologico-culturali e socio-economiche", che contraddistingue tanto le sue prestazioni quanto il suo assetto, cercando di pervenire alla ridefinizione dell'identità dell'avvocatura come "istituzione costituzionalmente qualificata". In questa chiave, ha rappresentato i rapporti ("costituzionalmente rilevanti") dell'avvocatura con la magistratura nel quadro di una situazione di crisi che mette in discussione la stessa legittimità dei due mondi. Lo studioso ha correttamente rimarcato che l'evoluzione del ceto forense non può essere valutata, anche in prospettiva futura, che nel quadro dell'Unione Europea, giacché col Trattato di Maastricht ha sostenuto - si è compiuto il processo $\mathrm{cd}$. di nation building iniziato in Italia nel 1861. Ha infine segnalato il fallimento delle strategie di modernizzazione del sistema giudiziario e l'illusorietà, da parte del ceto forense, sia della difesa corporativa a oltranza, sia della "logica aziendalistica", pronunciandosi a favore di una politica di eccellenza e di affidabilità dei professionisti dal punto di vista tecnico-giuridico.

Esaurito il compito di descrivere brevemente i dati più rilevanti usciti dalla ricerca, con sacrificio di molte altre considerazioni possibili, non credo di dover spendere molte parole di conclusione, almeno a questo stadio delle mie riflessioni. Lo impedisce, per paradosso, anche il tempo trascorso da quando l'indagine ha avuto termine effettivo. $\mathrm{Nel}$ frattempo, infatti, è cambiato il quadro esterno. La politica italiana, che per alcuni anni si è trascinata dietro asperrimi conflitti concernenti proprio il mondo della giustizia, è sembrata assumere repentinamente un altro corso che, si sperava, avrebbe almeno permesso di discutere di una crisi secolare con maggiore pacatezza e avendo presenti soprattut- 
to gli interessi della nostra debole democrazia. Sinora, questa speranza si è rivelata solo in parte fondata. Le vicende recenti dei progetti di legge sulla corruzione politica e sulle intercettazioni telefoniche mostrano che nessuno ha deposto le armi, pur in un quadro generale che esigerebbe la rinascita di quello spirito costituente che permise la rifondazione dello Stato nel "magico" periodo di gestazione della Carta fondamentale della Repubblica: da cui possono essere tratti utili insegnamenti per affrontare, fra le altre, anche le questioni affrontate nella ricerca qui presentata.

Una ulteriore riflessione è quindi doverosa e, nelle intenzioni di chi scrive, seguirà in tempi (si spera) meno lunghi di quelli decorsi tra la fine della ricerca e questo scritto. Già ora tuttavia credo di poter affacciare alcune ipotesi di lettura di questi dati e di quelli che cercherò di ricavare per aggiornarli nei punti essenziali. Si tratta del resto di osservazioni che ho già fatto in altre sedi e che, almeno finora, mi sono parse significative.

La prima di queste deriva da una constatazione che raramente si fa nelle discussioni sulla giustizia: la crisi italiana è particolarmente grave, ma non è una prerogativa esclusiva del nostro paese. Dieci anni $\mathrm{fa}$, recensendo un volume contenente gli atti di un convegno sulle istituzioni giudiziarie, svoltosi a Tokyo nel $2001,{ }^{38}$ mettevo in luce questo punto segnalando che la domanda di giustizia era cresciuta in tutti i paesi là rappresentati, con le conseguenti lentezze, quantunque si sappia bene che esistono differenze sensibili sul fronte della risposta del sistema. Uno sguardo fuori dai nostri confini permetterebbe di sfuggire alle emozioni del quotidiano, sovente suscitate da media che riferiscono di casi eclatanti assunti come regola piuttosto che come eccezione. Come dimostrato nella ricerca di Ghezzi e Quiroz Vitale, la mediatizzazione del tema della giustizia è determinante nell'orientare l'opinione pubblica: e un sistema di media formalmente libero, ma in realtà molto dipendente dalle lusinghe del potere (da cui provengono, direttamente o indirettamente, molti dei finanziamenti che gli permettono di vivere) dimentica troppo facilmente la regoletta giornalistica secondo cui è "notizia" quella che riferisce di un fatto imprevedibile ("non il

38 AA.VV. The Role of the Judiciary in Changing Societies. Edited by the Japanese Association of Sociology of Law, Kyoto, Kyoto University, 2001 (la recensione è apparsa in Sociologia del diritto, XXIX, 2002, pp. 167-170. 
cane che morde l'uomo, ma l'uomo che morde il cane", come si dice ai giovani redattori), non quanto avviene giornalmente, soprattutto se positivo per la collettività: le notizie buone, come noto, vengono normalmente ignorate perché non fanno impressione.

Con ciò non intendo dire che la giustizia italiana, affidata a un corpo di funzionari selezionato su basi tecnico-giuridiche, articolato al suo interno ma coeso e quasi isolato dal mondo esterno, funzioni molto meglio di quel che la gente pensa e, purtroppo, esperisce sulla propria pelle. Tutt'altro, ma le generalizzazioni sono pericolose perché inducono a intervenire in modo irrazionale o peggio emotivo, alla luce di qualche evento sensazionale. Così, oso dire, ha fatto il legislatore italiano nell'ambito della giustizia civile su cui si è abbattuta, e continua ad abbattersi, una serie di provvedimenti normativi, magari ben pensati dalle commissioni preparatorie, ma stravolti dal Parlamento, che non hanno risolto un solo problema di efficienza e in compenso hanno aumentato il tasso di incertezza del diritto persino su questioni che dovrebbero essere auto-evidenti come il calcolo dei termini processuali o la competenza dei singoli uffici giudiziari. Per contro, si sono sempre di fatto evasi i temi più banali, quali la copertura degli organici, le risorse da destinare alla giustizia, la preparazione del personale ausiliario, la ristrutturazione degli uffici secondo parametri organizzativi aggiornati: su questo punto, molto può essere fatto anche con le poche risorse a disposizione. ${ }^{39}$

Ma non è questo il punto principale che vorrei mettere sul tappeto per riflessioni future. Più significativo è un tema che è stato posto autorevolmente al centro delle discussioni sulla giustizia da Marc Galanter, notissimo specialista americano di disputing, e da altri autori, che nel 2004 hanno divulgato i dati più recenti della giustizia civile e penale negli Stati Uniti, rivelando come il processo tradizionale, mille volte rappresentato nei film, che termina col verdetto di una giuria e/o una decisione giudiziale dirimente, sempre minoritario nella storia di quel paese, sia ormai ridotto a un rango marginale: stabilmente e di molto sotto il $10 \%$ nei circuiti federali, poco sopra a livello statale. ${ }^{40}$

39 Come rivela il Bilancio di Responsabilità Sociale 2011 - Il Tribunale al servizio della comunità, pubblicato dal Tribunale di Milano nell'anno 2012.

40 Mi riferisco al numero speciale del Journal of Empirical Legal Studies, 1/3, nov. 2004 (che ho recensito in Sociologia del diritto, XXXIII, 2006, 1, pp. 207-209) e in 
Confesso che questi dati mi hanno procurato una viva impressione e mi hanno indotto a pensare, a titolo di ipotesi, che il sistema americano, recepito più o meno fedelmente in altri ordinamenti nel campo penale, regga solo perché ha elaborato alternative istituzionali o para-istituzionali che permettono di risolvere - e non in tempi brevi - controversie e processi: le prime, oggetto costante di negoziazione fra le parti (e spesso di abbandono), i secondi oggetto regolare di patteggiamento (plea bargaining) in un sistema che, per giunta, non obbliga il public prosecutor ad esercitare sempre l'azione penale.

Come è venuta a determinarsi questa realtà, che chiaramente tradisce un modello di giustizia fondato sulla sacralizzazione proprio di quella figura di giudice quivis de populo evocata da Ghezzi e Quiroz Vitale? Gli alti costi della giustizia, che gravano in modo particolarmente pesante sulle impugnazioni? Il rischio spesso incalcolabile della decisione delle giurie? La consapevolezza, nel processo penale, che la sentenza di primo grado è praticamente decisiva e immediatamente esecutiva? La sensazione, in generale, che il processo con giuria sia un lusso che solo le parti molto abbienti - O. J. Simpson, Michael Jackson - possono permettersi e che è di fatto precluso alla gente comune? Forse, da tutte queste considerazioni assieme, ben note alla pubblicistica statunitense, ma meno considerate negli altri paesi di quanto sarebbe opportuno.

Si può dunque dire che i sistemi di giustizia adottati nella modernità non collassano soltanto quando disapplicano i principi su cui si fonda? $\grave{E}$ un quesito imbarazzante che forse va posto una volta per tutte sul tavolo delle discussioni.

Per condurci dove, è un altro quesito, altrettanto delicato. Ghezzi indica la via più radicale, quella della dissoluzione della giustizia statale - per non dire dello stato stesso, forse dimenticando che per "stato" s'intende solo e semplicemente l'organizzazione istituzionale della polis, aperta o chiusa, minimalistica o dirigistica, democratica o autocratica, che è sempre esistita dacché esiste il genere umano. Ma non offre alternative, se non l'idea del giudice quivis de populo, di cui proprio l'esperienza americana mostra la debolezza intrinseca. Per non dire che un giudice che in nome del popolo esercita poteri ampiamente discrezio-

particolare all'accurato studio longitudinale di Galanter M. The Vanishing Trial: $A n$ Examination of Trials and Related Matters in Federal and State Courts. pp. 459 e ss. 
nali - come auspica Quiroz Vitale - evoca immagini troppo legate ad esperienze totalitarie di vario colore per non preoccupare.

Dobbiamo allora accontentarci, più umilmente, di interventi riformatori settoriali, in nome del principio di rango costituzionale che lo Stato è garante dei diritti dei cittadini? Forse, ma a patto che, come detto, siano elaborati con cura, inseriti coerentemente in un contesto normativo senza sconvolgerlo, attuati senza tradirne la logica intrinseca, avendo in mente un obiettivo di lungo periodo, non quello contingente di tranquillizzare l'opinione pubblica per qualche evento drammatico o, peggio, di salvare uno o più imputati dalle conseguenze di un reato.

Per interventi di questo tipo però occorre tempo e frattanto il collasso si aggrava. Per paradosso non è quindi da escludere che per rimettere il sistema sui binari siano necessari interventi eccezionali, perfino un'amnistia per i reati minori. Qualcuno ha mai pensato che il processo penale inquisitorio reggeva, e con fatica, soltanto perché ogni tre anni un'amnistia mandava in archivio decine di migliaia di processi penali che affollavano tutti i gradi di giudizio? Venute meno le amnistie, si è affacciato un surrogato - in sociologia si dice un equivalente funzionale - che produce effetti analoghi, sebbene su una scala meno egualitaria, giacché la prescrizione è spesso l'effetto di difese agguerrite che non tutti possono permettersi.

Queste - ripeto - sono libere considerazioni a margine di una ricerca che ancora suscita interrogativi più che offrire soluzione ai problemi su cui si è svolta. E sono altamente provvisorie. Le eventuali reazioni dei lettori saranno preziose per approfondirle, o cambiarle addirittura. Più vado avanti, più l'idea popperiana della unended quest mi sembra l'unica seria etica che uno studioso possa praticare. 